\title{
Three-Dimensional Structure in a Supersonic Jet: Behavior Near Centerline
}

S.H. Shih and R. Hixon

Institute for Computational Mechanics in Propulsion

Lewis Research Center

Cleveland, Ohio

Reda R. Mankbadi

National Aeronautics and Space Administration

Lewis Research Center Cleveland, Ohio

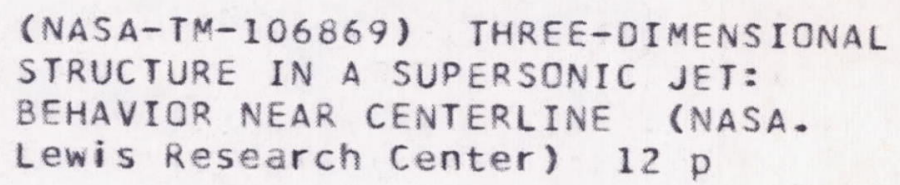

Prepared for the

33rd Aerospace Sciences Meeting and Exhibit sponsored by the American Institute of Aeronautics and Astronautics Reno, Nevada, January 9-12, 1995

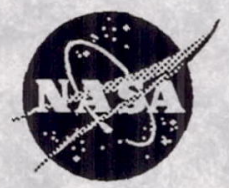

National Aeronautics and

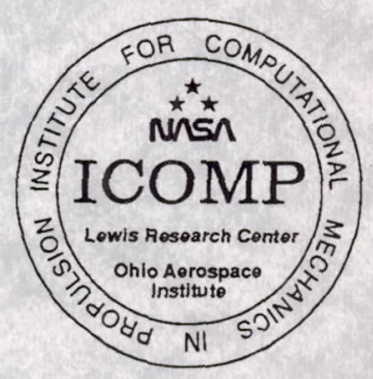




\title{
Three Dimensional Structure in a Supersonic Jet: Behavior Near Centerline
}

\author{
S. H. Shih* and D. R. Hixon* \\ Institute for Computational Mechanics in Propulsion \\ NASA Lewis Research Center \\ Cleveland, Ohio 44135 \\ R. R. Mankbadi** \\ NASA Lewis Research Center \\ Cleveland, Ohio 44135
}

\begin{abstract}
The unsteady structure of a supersonic jet is highly three dimensional, though the mean flow is axisymmetric. In simulating a circular jet the centerline represents a computational boundary. As such, spurious modes can be generated near centerline, unless special attention is given to the behavior of the 3D structure near the centerline. Improper treatment of the dependent variables near the centerline results in the solution diverging or being suitable only for small amplitude excitation. With a careful treatment of the centerline formulation, no spurious mode is generated. The results show that a near linear disturbance growth is obtained, as the linear stability theory indicates. At high levels of excitation, nonlinear development of disturbances is evident and saturation is reached downstream.
\end{abstract}

\section{Introduction}

Jet noise suppression has become a critical issue for the development of high speed civil transport plane. The jet noise is generated by the time dependent flow fluctuations in the near field which are associated with pressure fluctuations that propagate to the far field producing the radiated sound. Experiments have shown that the measured sound fields appear to emanate from a region about 10 diameters downstream of the nozzle exit [1]. This noise-producing initial region of the jet is characterized by a large scale vortical structure and can be viewed as having a wavelike nature. It is believed that the large scale structure is more efficient than the small scale structure in radiating sound [1-5]. This indicates that the initial development of the jet should be clearly resolved

* Senior research associate, Member AIAA

** Senior scientist and technical leader, CAA, Associate fellow AIAA so that an accurate noise prediction can be made. The near flow field is described by the unsteady NavierStokes equations. However, direct numerical simulation can not resolve all scales of motion for high Reynolds number flows. It is appropriate to perform large-eddy simulations to accurately capture the large scales of motion while modelling the sub-grid scale turbulence.

The use of large-eddy simulations (LES) as a tool for prediction of the jet noise source has been proposed by Mankbadi et al. [6]. Not only the mean flow must be calculated accurately, but also the physical flow fluctuations must be accurately predicted since the sound source is given in terms of the flow fluctuations. Since the computational domain is usually finite, the numerical boundary conditions can generate spurious modes that render the computed flow fluctuations totally unacceptable. In simulating a circular jet, the centerline $(r=0)$ represents a computational boundary. Boundary condition for axisymmetric mean flow is obvious. However, the unsteady structure of a supersonic jet is highly three dimensional, even though the mean flow is axisymmetric (see, for instance, Mankbadi [5], Michalke [7]). As such, spurious modes can be generated near the centerline, unless special attention is given to the behavior of the three dimensional structure near the centerline, which is the subject of the present work.

The numerical solution of the Navier-Stokes equations in cylindrical coordinates requires the proper treatment of the discretized equations at the centerline. In axisymmetric jet flow, Mankbadi et al [6] derived a new set of equations at the centerline from the original equations using L'Hospitals rule to circumvent numerical problems associated with the geometric singularity in the formulation. In the present study, three approaches to the centerline treatment are considered, namely, asymptotic, averaging and interior points approaches. The effect of sub-grid scale turbulence stresses is not taken into account in this study to avoid any uncertainty from the uir- 
bulence models. A supersonic jet with Mach number = 1.5, Reynolds number $=1.27 \times 10^{6}$ based on the nozzle exit diameter and jet centerline parameters is considered. The outer stream is 0.25 of the jet exit velocity, and the jet temperature ratio is 0.5 . Time-harmonic disturbances are imposed at the inflow boundary of the jet, and the subsequent development of disturbances are examined.

\section{Governing Eauations}

The flow field of a supersonic jet is governed by the compressible Navier-Stokes equations, which can be written in cylindrical coordinates as

$\frac{\partial Q}{\partial t}+\frac{\partial F}{\partial x}+\frac{1}{r} \frac{\partial}{\partial r}(r G)+\frac{1}{r} \frac{\partial H}{\partial \phi}=S$

where

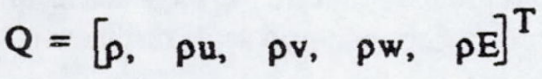

$F=\left[\begin{array}{c}\rho u \\ p+\rho u^{2}-\sigma_{x x} \\ \rho u v-\sigma_{x x} \\ \rho u w-\sigma_{x \phi} \\ \rho u I-u \sigma_{x x}-v \sigma_{x x}-w \sigma_{x \varphi}-k \frac{\partial T}{\partial x}\end{array}\right]$

$G=\left[\begin{array}{c}\rho v \\ \rho u v-\sigma_{x r} \\ p+\rho v^{2}-\sigma_{r r} \\ \rho v w-\sigma_{r \phi} \\ \rho v I-u \sigma_{x r}-v \sigma_{r r}-w \sigma_{r \phi}-k \frac{\partial T}{\partial r}\end{array}\right]$

$H=\left[\begin{array}{c}\rho w \\ \rho u w-\sigma_{x \phi} \\ \rho v w-\sigma_{r \phi} \\ p+\rho w^{2}-\sigma_{\phi \phi} \\ \rho w I-u \sigma_{x \phi}-v \sigma_{r \phi}-w \sigma_{\phi \phi}-k \frac{\partial T}{\partial \phi}\end{array}\right]$

$$
S=\frac{1}{r}\left[\begin{array}{c}
0 \\
0 \\
p+\rho w^{2}-\sigma_{\phi \phi} \\
-\rho v w+\sigma_{r \phi} \\
0
\end{array}\right]
$$

Here $Q$ is the unknown vector, F, G, and $H$ are the fluxes in the $x, r$, and $\phi$ directions, respectively; $S$ is the source term that arises in cylindrical polar coordinates; and $\mathrm{k}$ is thermal conductivity. The total enthalpy is I, the total energy is $\mathrm{E}$, and $\sigma_{\mathrm{ij}}$ are the viscous stresses. This system of equations is coupled with the equation of state for a perfect gas.

\section{Numerical Scheme}

The importance of the dispersion and dissipation of a given scheme, which used in connection with computational aeroacoustics, were highlighted by Hardin [8]. Both effects are crucial in computational seroacoustics, and can render the computed unsteady part of the solution completely unacceptable. As such, high-order accurate schemes are required for problems in computational aeroacoustics.

A fourth-order accurate in space, second-order accurate in time scheme is used, which is an extension of the MacCormack scheme by Gottlieb and Turkel [9]. Mankbadi et al. [6] used this scheme to study the structure of axisymmetric supersonic jet flow and its radiated sound. Ragab and Sheen [10], and Farouk, Oran and Kailasanath [11] have also successfully applied this scheme for the study of nonlinear instability problems in plane shear layers. Sankar, Reddy and Hariharan [12] performed a comparative study of various numerical schernes for aeroacoustics applications, and found that this scheme offers high spatial accuracy. In this scheme, the operator is split into three one-dimensional operators and applied in a symmetric way to avoid biasing of the solution:

$\mathrm{Q}^{\mathrm{n}+2}=\mathrm{L}_{2 \mathrm{x}} \mathrm{L}_{2 \mathrm{r}} \mathrm{L}_{2 \phi} \mathrm{L}_{1 \phi} \mathrm{L}_{1 \mathrm{r}} \mathrm{L}_{1 \mathrm{x}} \mathrm{Q}^{\mathrm{n}}$

where $L$ represents the one-dimensional operator. Each operator consists of a predictor and a corrector steps, and each step uses one-sided differencing:

Predictor:

$$
Q_{i}^{n+\frac{1}{2}}=Q_{i}^{n}-\frac{\Delta t}{6 \Delta x}\left(7 F_{i}-8 F_{i-1}+F_{i-2}\right)^{n}
$$

Corrector: 


$$
\begin{aligned}
& Q_{i}^{n+1}=\frac{1}{2}\left[Q_{i}^{n}+Q_{i}^{n+\frac{1}{2}}\right. \\
& \left.+\frac{\Delta t}{6 \Delta x}\left(7 F_{i}-8 F_{i-1}+F_{i-2}\right)^{n+\frac{1}{2}}\right]
\end{aligned}
$$

and likewise for the radial and azimuthal directions. The scheme becomes fourth-order accurate in the spatial derivatives when alternated with symmetrical variants. Let $\mathrm{L}_{1}$ be the one-dimensional operator with forward difference in the predictor and backward difference in the corrector, then $L_{2}$ will be the one-dimensional operator with backward difference in the predictor and forward difference in the corrector.

\section{Boundary Conditions}

The scheme uses one-sided differences for the fluxes. A cubic extrapolation is used to obtain the fluxes at two ghost points outside the computational domain in order to update the boundary points, i.e.

$$
\begin{aligned}
& F_{m+1}=4 F_{m}-6 F_{m-1}+4 F_{m-2}-F_{m-3} \\
& F_{m+2}=4 F_{m+1}-6 F_{m}+4 F_{m-1}-F_{m-2}
\end{aligned}
$$

The physical outflow boundary conditions for the computation are derived using linearized characteristics $[13,14]$ to permit the unsteady flow properties to pass without producing non-physical reflections. A summary of the outflow boundary conditions is given in Mankbadi et al. [6].

The inflow was taken to be the mean flow with a hyperbolic-tangent profile plus time-harmonic disturbances. The initial mean axial velocity profile is given by Michalke and Hermann [15] as

$$
\frac{u-u_{0}}{\frac{1}{2}\left(u_{j}-u_{0}\right)}=1-\tanh \left[\frac{1}{4 \theta}\left(r-\frac{1}{r}\right)\right]
$$

where $u_{j}$ is the exit velocity of the jet, $u_{0}$ denotes the velocity of coflow and $\theta$ is the momentum boundary layer thickness of the jet shear layer. The momentum thickness $\theta$ and radius $r$ are normalized by the nozzle exit radius $R$. The corresponding temperature is specified by the Crocco's relation, which can be expressed as

$$
\begin{gathered}
T=T_{0}+\left(T_{j}-T_{0}\right) \cdot \frac{\left(u-u_{0}\right)}{\left.u_{j}-u_{0}\right)}+0.5 T_{j}(\gamma-1) M^{2} \\
\cdot\left(u_{j}-u\right) \frac{\left(u-u_{0}\right)}{\left(u_{j}-u_{0}\right)^{2}}
\end{gathered}
$$

where $M$ is the Mach number. The time-harmonic disturbances consist of a single, symmetrical pair of the first helical mode, i.e.

$\left[u^{\prime} v^{\prime} w^{\prime} p^{\prime} \rho^{\prime}\right]=\Phi(r) e^{i(\alpha x-\omega t)} \cos (\phi)+C \alpha(14)$ where $\alpha$ is the wavenumber and $C C$ is the complex conjugate. The angular frequency $\omega$ is chosen such that the corresponding Strouhal number $S t=f(2 R) / u_{j}=0.125$. The eigenfunctions $\Phi(r)$ are obtained from the solution of linear stability equations for velocities, density, and pressure respectively.

$\Phi(r)=[\hat{u}(r) \hat{v}(r) \hat{w}(r) \hat{p}(r) \hat{\rho}(r)]$

We now focus our attention on the flow behavior near the centerline, as $r$ approaches zero.

\section{Centerline Formulations}

In implementing the fourth-order scheme near the centerline, two ghost points will be needed for updating. We found that using two ghost points near the centerline generates nonphysical oscillations and causes the code to blow up. Instead, we used the second-order MacCormack scheme for updating this point. Therefore, only one ghost point is needed. Three approaches to centerline treatment are considered and discussed below.

\subsection{Assmptotic Beharior ol Navier-Stokes Equations Near the Centerline}

Starting from the Navier-Stokes equations, and requiring that the flow variables should be finite for $r=0$, we find that the azimuthal velocity $w$ must satisfy the differential equation

$$
\frac{\partial^{2} w}{\partial \phi^{2}}+w=0
$$

The solution for w near the centerline can thus be written as

$\mathbf{w}=\mathrm{A} \cos \phi+\mathrm{B} \sin \phi$

We chose to study a top-bottom symmetrical jet, i.e. the three-dimensional helical modes come in pairs as the ex- 
perimental investigation of Cohen and Wygnanski [16], and the theoretical investigation of Mankbadi $[5,16]$ have shown. In this case, the constant $A$ in the above equation is set to zero. The formulations for $u, v, p$ and $p$ can be derived in the same way. It follows from the Navier-Stokes equations that the flow behavior at $r=0$ can be written as

$$
\begin{aligned}
& w(\phi)=-v\left(\phi_{0}\right) \frac{\sin \phi}{\cos \phi_{0}} \\
& \frac{\partial}{\partial r} u(\phi)=\frac{\partial}{\partial r} u\left(\phi_{0}\right) \frac{\cos \phi}{\cos \phi_{0}} \\
& v(\phi)=v\left(\phi_{0}\right) \cdot \frac{\cos \phi}{\cos \phi_{0}} \\
& \frac{\partial}{\partial r} p(\phi)=\frac{\partial}{\partial r} p\left(\phi_{0}\right) \frac{\cos \phi_{0}}{\cos \phi_{0}} \\
& \frac{\partial}{\partial r} \rho(\phi)=\frac{\partial}{\partial r} \rho\left(\phi_{0}\right) \frac{\cos \phi_{0}}{\cos \phi_{0}}
\end{aligned}
$$

These equations are valid at $r=r_{0}$ where $r_{0}$ is a small quantity and is taken to be 0.01 in the present study. Thus the above equations describe the solution at $\phi$ on the circle $r=r_{0}$ in terms of the solution at $\phi_{0}$. In implementing this condition, the solution at $\phi_{0}$ is obtained by taking the average of the values at $0^{\circ}$ and $180^{\circ}$.

\section{Averaging}

Another alternative that can be used is the simple averag. ing. The idea is that the flow variable at centerline $r=0$ is single-valued and independent of the azimuthal direction. In implementing this approach, the flow variables $u$, $v, w, p$, and $p$ at $r=0$ are taken to be the azimuthal average of all points at $r=\Delta r$.

\subsection{Centerline as an interior point}

The centerline boundary condition is in fact an artificial one that arises from the fact that polar coordinates are used with r starting from zero. Imagine performing full jet simulation with $r$ extending from $-\infty$ to $\infty$, thus point " $\mathrm{a}$ " closest to the centerline (figure 1) can be treated as an interior point. If a second-order scheme is used, then the flux at point " $b$ " is needed for updating point " $a$ ". Since we are solving half a jet, point " $\mathrm{b}$ " is a ghost point. But because of symmetry, the flux at point " $b$ " can be related to that of the interior point " $\mathrm{c}$ " as follows

$$
\begin{aligned}
& M_{b}=M_{c} \\
& N_{b}=-N_{c}
\end{aligned}
$$

where $M$ represents the mass, axial or radial momentum, or energy flux, while $\mathrm{N}$ is the momentum flux in the azimuthal direction.

\section{Results and Discussion}

A supersonic jet with Mach number 1.5, based on the jet exit parameters, is considered in this study. The velocity ratio $u_{d} / u_{j}$ is 0.25 , and the temperature $T_{d} / T_{j}$ is 0.5 . The Reynolds number based on the jet dianeter and jet exit centerline velocity is $1.27 \times 10^{6}$. The parameter, momentum thickness $\theta$, in the initial axial velocity profile is 0.125 . The inflow is taken to be the mean plus the time harmonic disturbance.

$Q=Q+\varepsilon \cdot Q^{\prime}$

where $\bar{Q}$ and $Q$ ' were given in the previous section, and $\varepsilon$ is the input excitation level. The computational domain (as shown in figure 2) extends 5 radii in the radial direction, and 50 radii in the axial direction. $\phi$ ranges from 0 to 180 degrees in the azimuthal direction, since we consider a top-bottom symmetrical jet, and only half of the circular jet is solved. The computational grid consists of $300 \times 100 \times 13$ mesh points in the axial, radial and azimuthal directions respectively. The mesh is uniform in the axial and azimuthal directions, and stretched in the radial direction with concentration of the grid points around $r=R$. The computations started with a time step corresponding to a CFL number of 0.3 , and later on, after the initial transient purged out of the computational domain, a finer time step was used to collect the computed data for use in Fourier transform.

Figure 3 shows a snap shot of the predicted density contours using the asymptotic formulation, and a clean behavior near the centerline is evident. The excitation level $\varepsilon$ is $1 \times 10^{-5}$ in this case. For the sake of comparison, figure 4 shows a snap shot of the density with no careful treatment of the centerline. These results were obtained using a second-order MacCormack scheme for the points at $r=r_{0}+\Delta r$, and second-order extrapolation for the points at $r=r_{\sigma}$. As indicated in figure 4, nonphysical values were generated near the centerline. Figure 5 shows the spectra at $r=r_{0}, x=40 R$. This figure shows that the amplitude peaks at the forcing frequency $S t=0.125$ and its harmon$i c$, and no spurious modes were generated when the asymptotic formulation is used. Without careful treatment of the centerline, spurious modes were produced that rendered the solution totally unacceptable.

Figure 6 shows the growth of the input axial velocity disturbance at $r / R=1$ for several excitation levels using 
the asymptotic formulation. The time span in which the data was collected is 16 characteristic times, where the characteristic time is defined as the ratio of nozzle exit radius to the jet centerline velocity. This time span corresponds to the period of the input disturbance when the Strouhal number is 0.125 . Fourier transforms are performed for the collected time dependent dat to obtain the amplitude of the disturbances at each location. As one can see in figure 6, the disturbance grows linearly, as linear stability theory indicates. At high levels of excitation, nonlinear effects occur and saturation is reached downstream.

Next, we show the results obtained using simple averaging at the jet centerline. A snap shot of the predicted density contours is shown in figure 7. Clean behavior near the centerline is obtained. Figure 8 presents the growth of the input axial velocity disturbances at $r / R=1$ for various excitation levels. An initial linear development of disturbances can be clearly seen and nonlinear effects take place when moving further downstream. The saturation of disturbance growth is also reached downstream.

Figure 9 shows a snap shot of the predicted density contours for $\varepsilon=1 \times 10^{-5}$ using the interior point approach. No nonphysical value is generated near the centerline except the region near the outflow boundary. Figure 10 presents the growth of the input axial velocity disturbances at $\mathrm{r} / \mathrm{R}=1$ for various excitation levels. The same phenomenon as those of the previous two approaches occurred, i.e. an initial linear growth of disturbance followed by its nonlinear development. The saturation of disturbance growth is also reached downstream. Figure 11 shows the comparison of the growth of axial velocity disturbances at $r / R=1$ using the three different approaches. The results obtained using the interior point approach have a slightly higher growth rate than the results obtained using the asymptotic and averaging approaches.

The three-dimensional structure of the flow field is shown in figures 12-14. Figure 12 shows the snap shot of the computed velocity vectors in $\mathrm{r}-\phi$ plane at $\mathrm{x}=28.3$, 33.3 and 38.3. As demonstrated in this figure, the cross flow velocity increases and changes its direction when the flow goes downstream; a clear evidence of the development of streamwise vorticities. Figure 13 shows the kinetic energy and vorticity contours at $\phi=0$ and 180 degrees. It is seen that the symmetry of the flow no longer exists downstream. Figure 14 shows the iso-surfaces for the kinetic energy and vorticity magnitude at time $t=100$. The helical nature of the structure and the roll-up of vortices are evident.

\section{Conclusions}

Direct simulations of a supersonic round jet flow field were presented with emphasis on the numerical treatment of the centerline $(r=0)$. Three approaches, asymptotic, averaging and interior point, were considered in the present study. Similar results were produced by all three approaches; a clean behavior of the flow near centerline, linear growth followed by nonlinear development of the disturbances, and the helical natural of the flow structure. Currently, attempts are under way to patch the computed nonlinear flow field to a linearized Euler solution to obtain the far-field sound.

\section{References}

1. Seiner, J. M., McLaughlin, D. K. and Liu, C. H., "Supersonic Jet Noise Generated by Large-Scale Instabilities," NASA TP-2072, September, 1982.

2. Zaman, K. B. M. Q., "Flow Field and Near and Far Sound Field of a Subsonic Jet," Journal of Sound and Vibration, Vol. 106, pp. 1-6, 1986.

3. Mankbadi, R. R. and Liu, J. T. C., "Sound Generated Aerodynamically Revisited: Large-Scale Structures in a Turbulent Jet as a Source of Sound," Philos. Trans. Royal Society of London A, Vol. 311, pp. 183-217, 1984.

4. Mankbadi, R. R., "The Self Noise from Ordered Structures in a Low Mach Number Jet, Journal of Applied Mechanics, Vol. 57, pp. 241-246, 1990.

5. Mankbadi, R. R., "Dynamics and Control of Coherent Structure in Turbulent Jets, ${ }^{\text {Applied Mechanics }}$ Reviews, Vol. 45, No. 6, pp. 219-247, 1992.

6. Mankbadi, R.R., Hayder, M.E. and Povinelli, L.A., "The Structure of Supersonic Jet Flow and Its Radiated Sound," AIAA Journal, Vol. 32, pp. 897-906, 1994.

7. Michalke, A., "Survey on Jet Instability Theory," Progress in Aerospace Science, Vol. 21, pp. 159. 199, 1984.

8. Hardin, J. C., "Recent Insights into Computational Aero-Acoustics," in Computational Aero- and Hydro-Acoustics, edited by R. R. Mankbadi, A. S. Lyrintzis, O. Baysal, L. A. Povinelli, M. Y. Hussaini, FED-Vol. 147, pp. 1, 1993.

9. Gottlieb, D. and Turkel, E., "Dissipative Two-Four Methods for Time-Dependent Problems," Mathematics of Computation, Vol. 30, No. 136, pp. 703$723,1976$.

10. Ragab, S. A. and Sheen, S., "The Nonlinear Development of Supersonic Instability Waves in a Mixing Layer," Physics of Fluids A, Vol. 4, pp. 553-566, 1991. 
11. Farouk, B., Oran, E. S. and Kailasanath, K., "Numerical Simulations of the Structure of Supersonic Shear Layers," Physics of Fluids A, Vol. 3, pp. 2786-2798, 1991.

12. Sankar, L. N., Reddy, N. N. and Hariharan, N., "A Comparative Study of Numerical Scheme for AeroAcoustic Applications," in Computational Aeroand Hydro-Acoustics, edited by R. R. Mankbadi, A. S. Lyrintzis, O. Baysal, L. A. Povinelli, M. Y. Hussaini, FED-Vol. 147, pp. 1, 1993.

13. Hayder, M. E., Turkel, E. and Mankbadi, R. R., "Numerical Simulation of a High Mach Number Jet," AIAA Paper 93-0653, 1993.
14. Bayliss, A. and Turkel, E., "Far Field Boundary Condition for Compressible Flows," Journal of Computational Physics, Vol. 48, pp. 182-199, 1982.

15. Michalke, A. and Hermann, G., "On the Inviscid Instability of a Circular Jet with Exrernal Flow," Journal of Fluid Mechanics, Vol. 114, pp. 343, 1982.

16. Cohan, J. and Wygnanski, I., "The Evolution of Instabilities in the Axisymmetric Jes, par 2, The Flow Resulting From the Interaction Between Two Waves," Journal of Fluid Mechanics, Vol. 176, pp. 121-235, 1987.

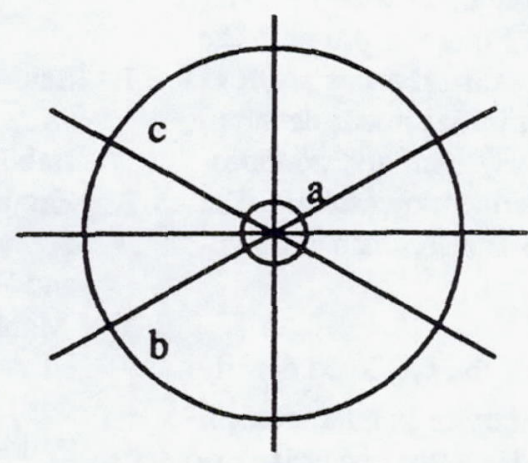

Fig. 1 Schematic of meshes near centerline, $r-\phi$ plane.

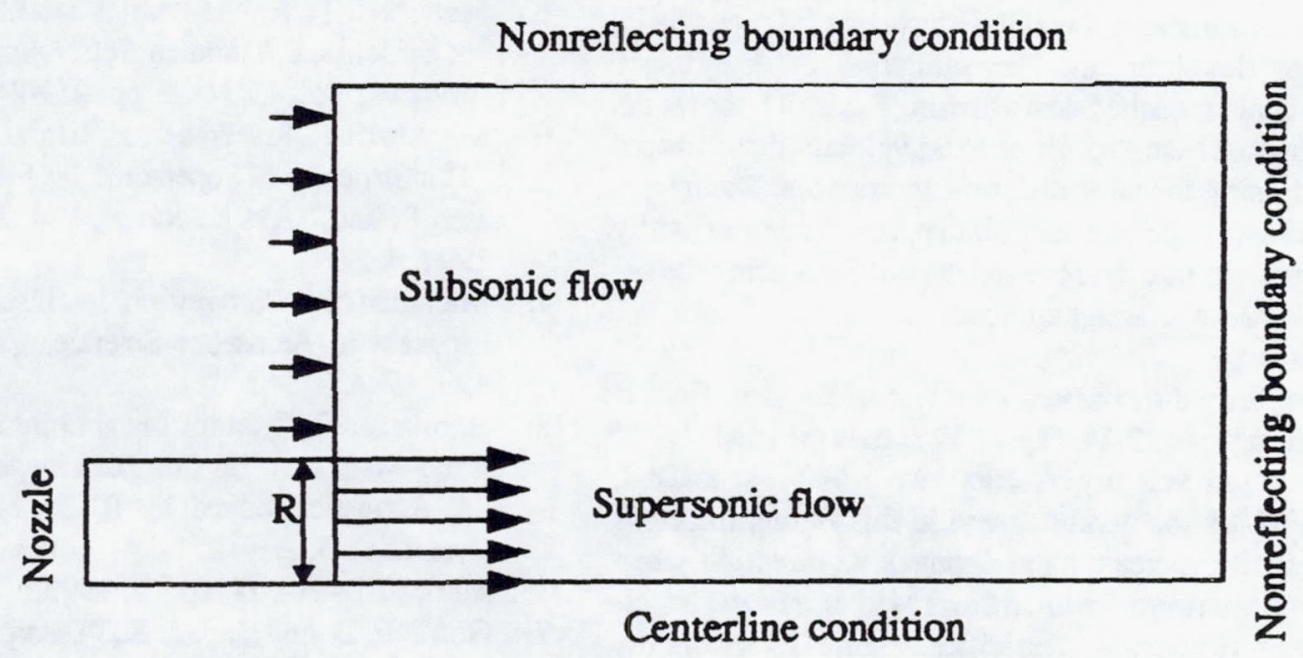

Fig. 2 Computational Domain 


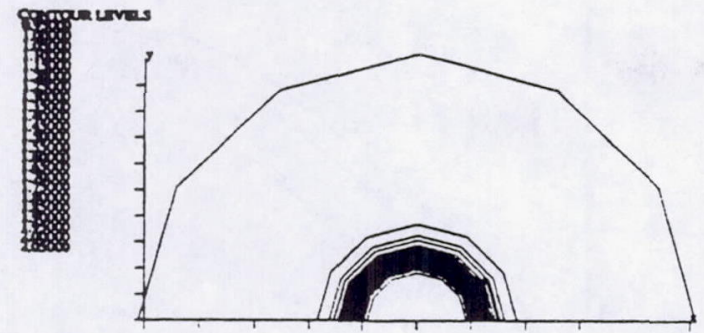

(a)

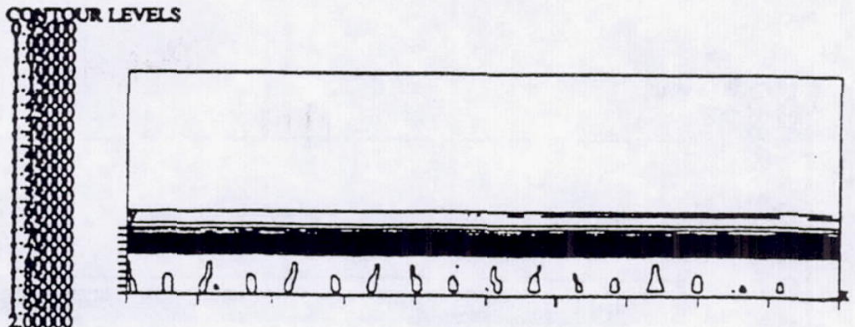

(b)

Fig. 3 Snap shot of the predicted density contours at $t=100$ using asymptotic formulation, (a) $r-\phi$ plane, $x=40$ (b) $x-r$ plane, $\phi=0$ deg.

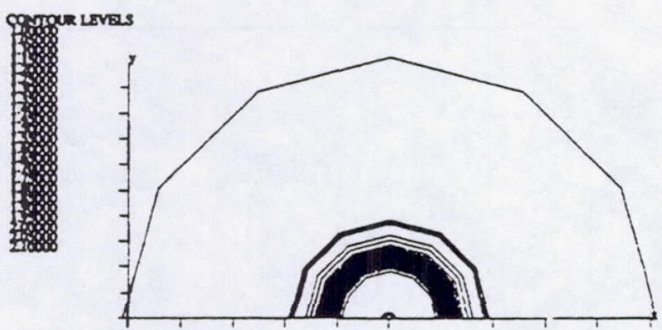

(a)

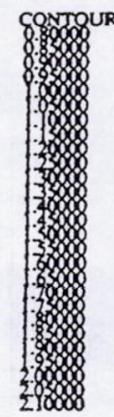

(b)

Fig.4 Snap shot of the predicted density contours at $t=100$ using extrapolation, (a) $r-\phi$ plane, $x=40$ (b) $x$-r plane, $\phi=0$ deg.

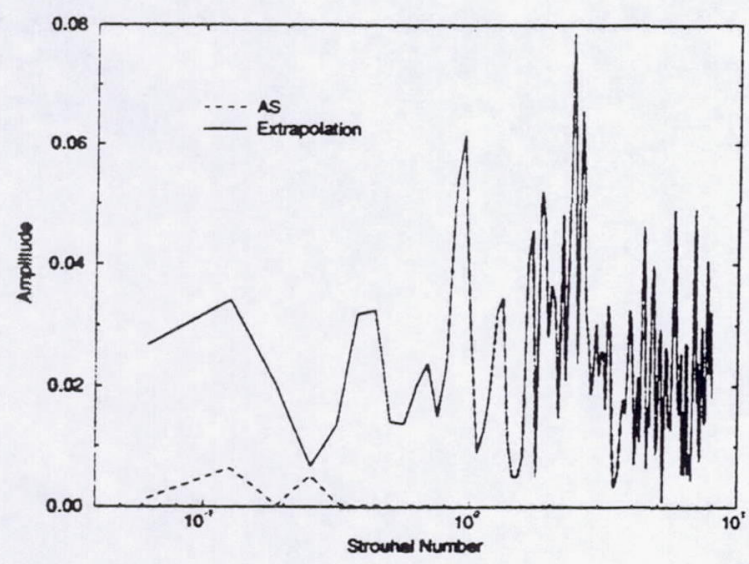

Fig. 5 Comparison of axial velocity spectra at the centerline, $x=40, \phi=0$ deg.

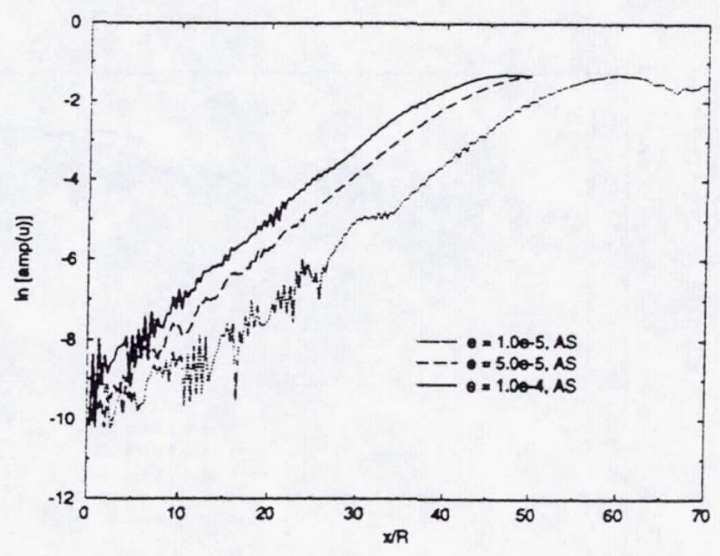

Fig. 6 The growth of axial velocity disturbance at $r=1$ using asymptotic formulation. 

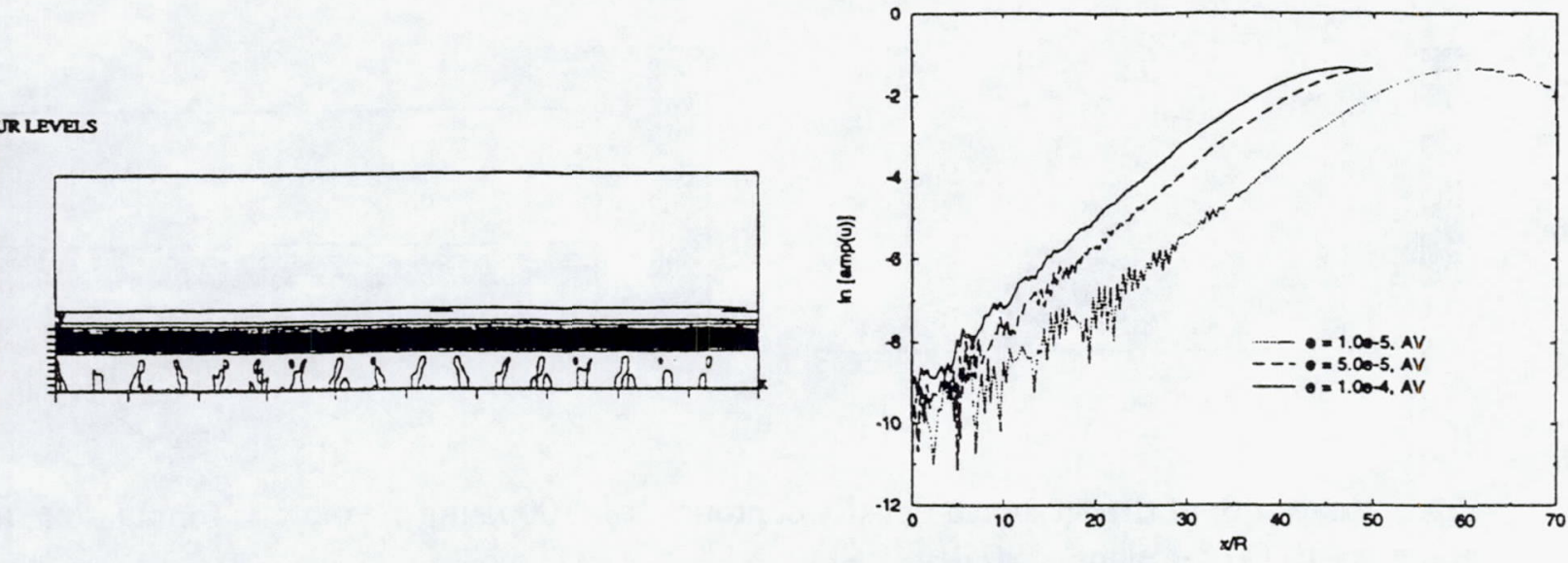

Fig. 7 Snap shot of the predicted density contours at $\mathrm{t}=100$ using averaging approach, $\mathrm{x}-\mathrm{r}$ plane, $\phi=0$ deg.

Fig. 8 The growth of axial velocity disturbance at $r=1$ using averaging approach.
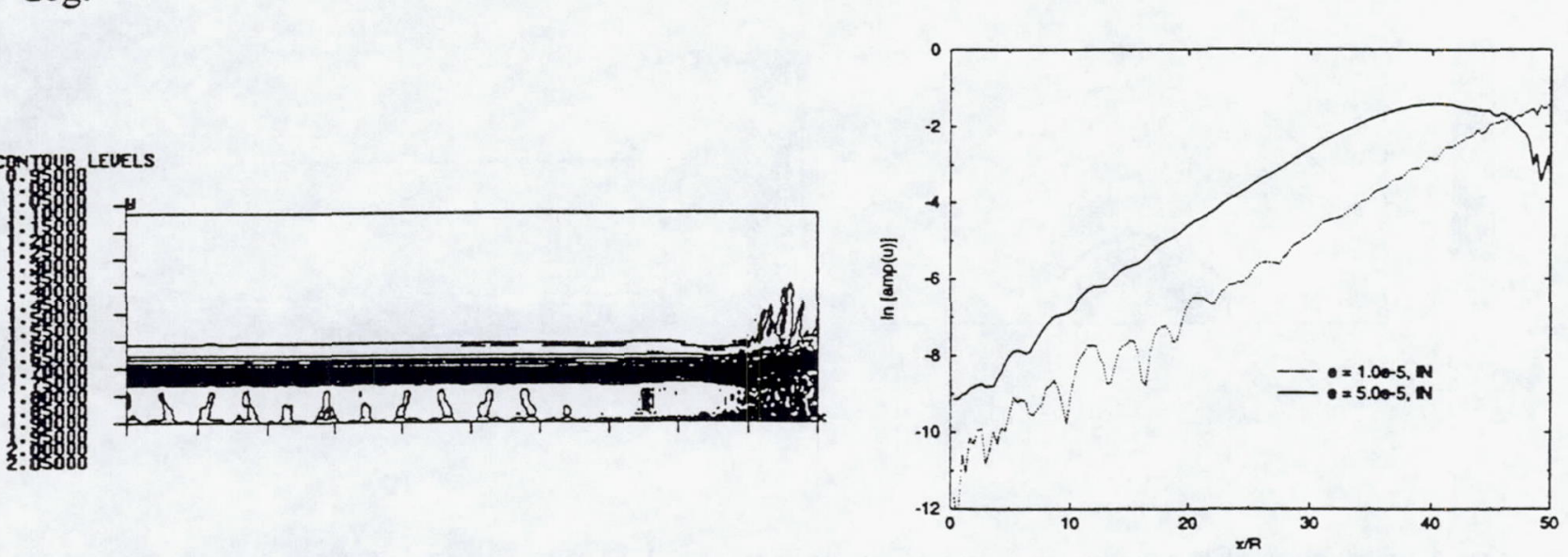

Fig. 9 Snap shot of the predicted density contours at $\mathrm{t}=100 \mathrm{using}$ interior point approach, $\mathrm{x}-\mathrm{r}$ plane, $\phi=0 \mathrm{deg}$.

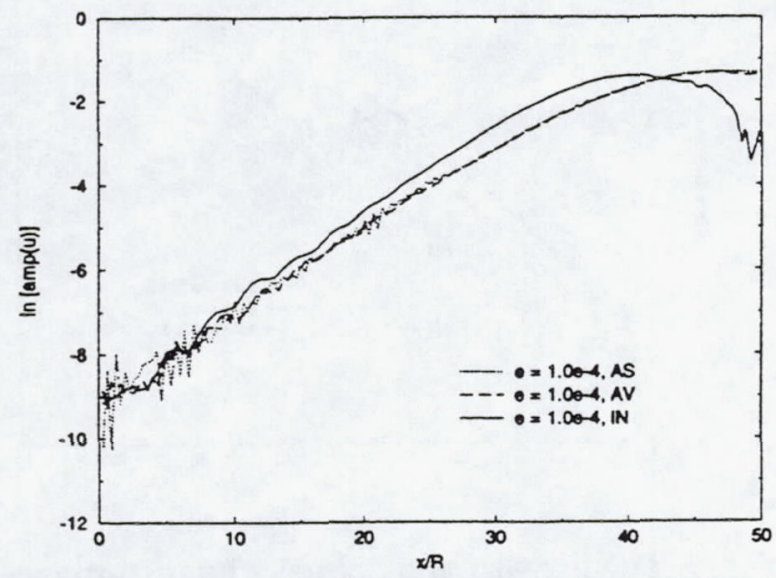

Fig. 11 Comparison of the growth of axial velocity disturbance at $\mathrm{r}=1$.

Fig. 10 The growth of axial velocity disturbance at $r=1$ using interior point approach. 


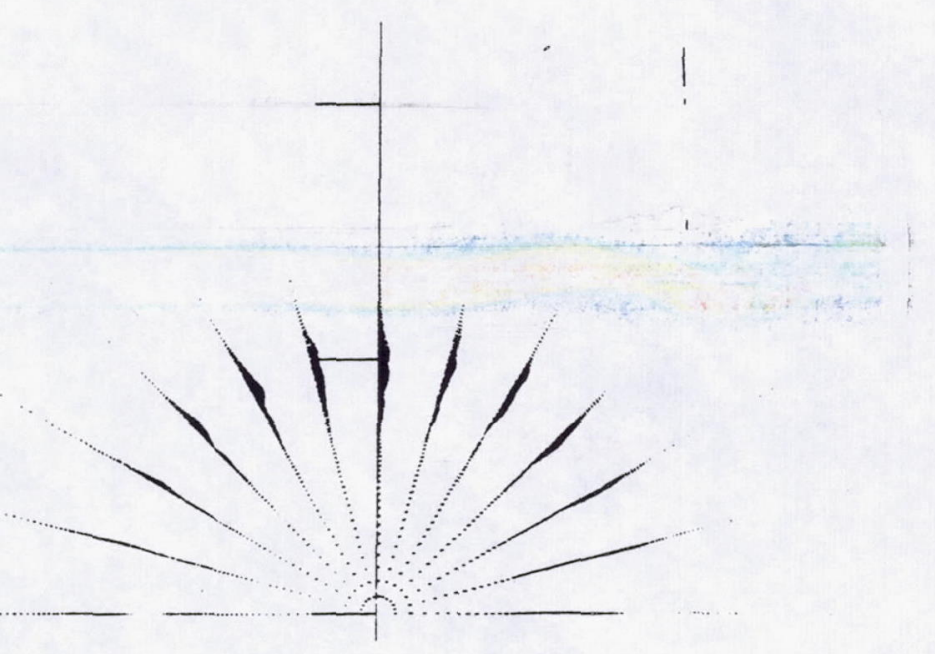

(a)

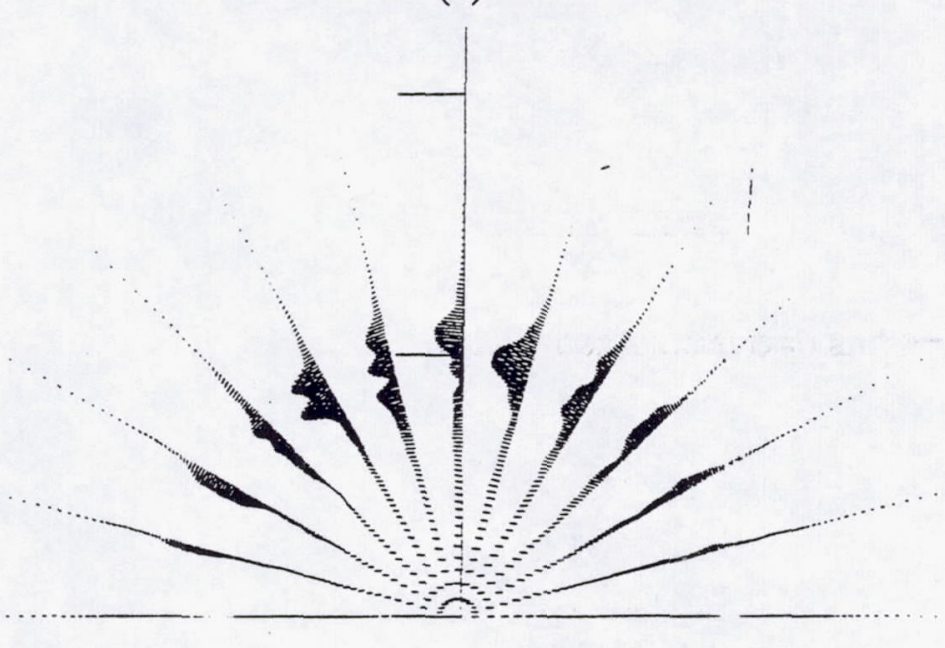

(b)

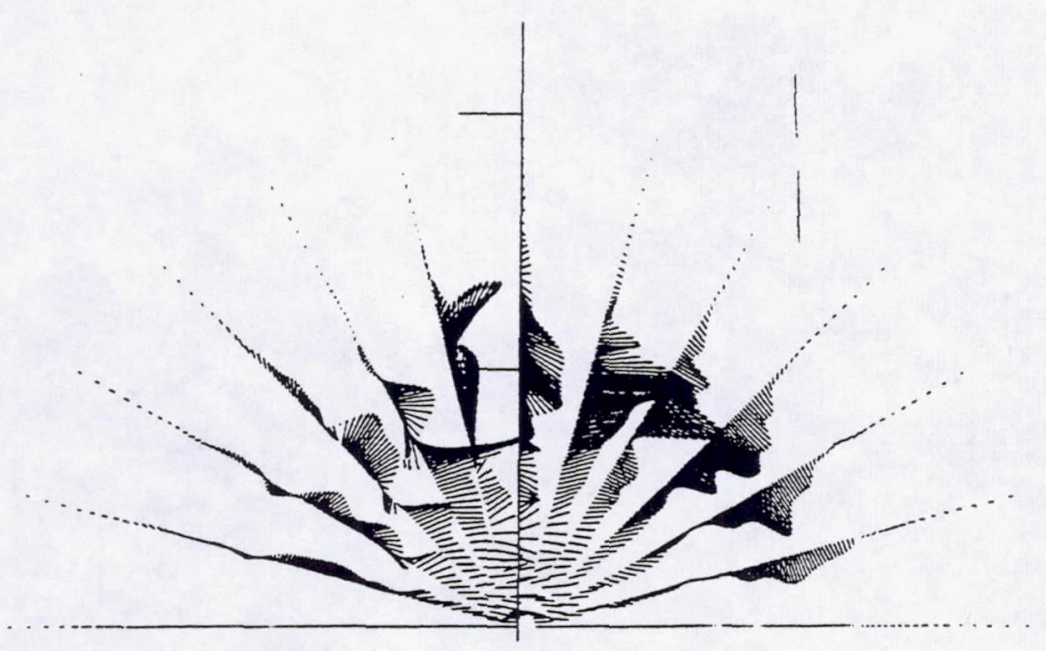

(c)

Fig. 12 Snap shot of the computed velocity vectors at $t=100$ in $r-\phi$ plane, (a) $x=28.3$ (b) $x=33.3$ (c) $x=38$. 3 . 
Page intentionally left blank 
(a)

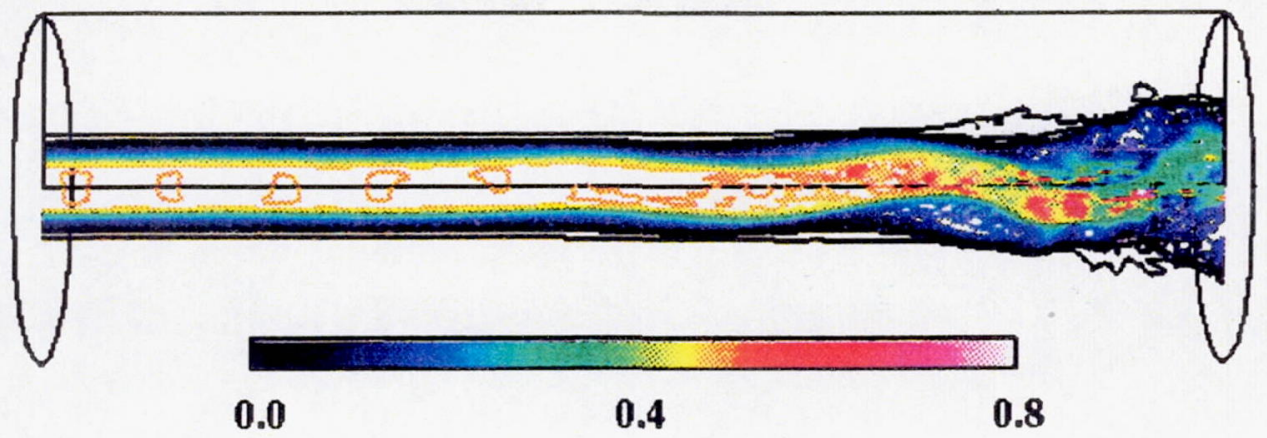

(b)
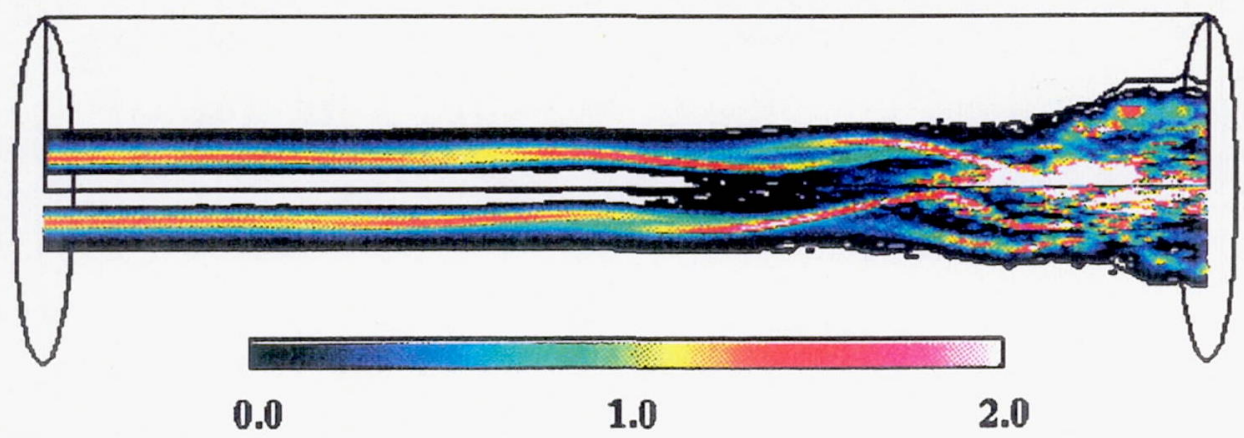

Fig. 13 Snap shot of the predicted kinetic energy and vorticity contours at $\phi=0$ and $\phi=180 \mathrm{deg}$., $\mathrm{t}=100$, (a) kinetic energy, (b) vorticity magnitude.

(a)

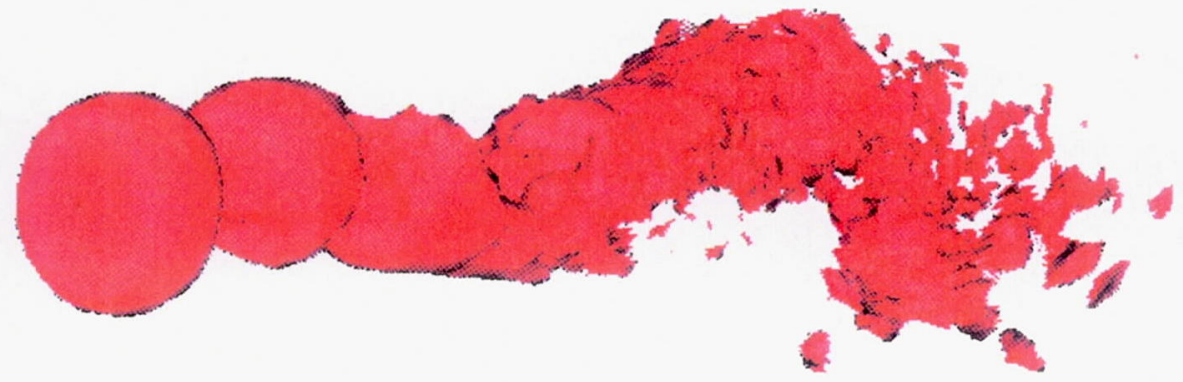

(b)

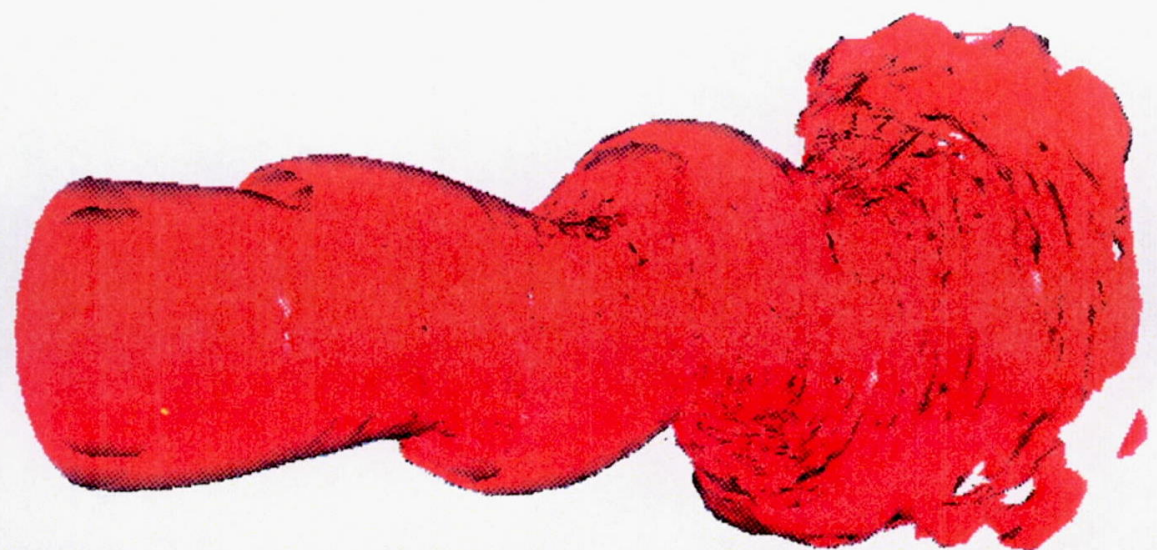

Fig. 14 Snap shot of the predicted kinetic energy and vorticity isosurfaces at $t=100$, (a) kinetic energy $=0.5$, (b) vorticity magnitude $=0.8$. 
Public reporting burden for this collection of information is estimated to average 1 hour per response, including the time for reviewing instructions. searching existing data sources, gathering and maintaining the data needed, and completing and reviewing the collection of information. Send comments regarding this burden estimate or any other aspect of this collection of information, including suggestions for reducing this burden, to Washington Headquarters Services, Directorate for Information Operations and Reports, 1215 Jefferson Davis Highway, Suite 1204, Arlington, VA 22202-4302, and to the Office of Management and Budget, Paperwork Reduction Project (0704-0188), Washington, DC 20503.

\begin{tabular}{|l|c|c|}
\hline 1. AGENCY USE ONLY (Leave blank) & $\begin{array}{c}\text { 2. REPORT DATE } \\
\text { March } 1995\end{array}$ & $\begin{array}{r}\text { 3. REPORT TYPE AND DATES COVERED } \\
\text { Technical Memorandum }\end{array}$
\end{tabular}

4. TITLE AND SUBTITLE

5. FUNDING NUMBERS

Three-Dimensional Structure in a Supersonic Jet: Behavior Near Centerline

6. AUTHOR(S)

S.H. Shih, D.R. Hixon, and R.R. Mankbadi

WU-505-90-5K

NCC3-370

8. PERFORMING ORGANIZATION

REPORT NUMBER

National Aeronautics and Space Administration

Lewis Research Center

E-9487

Cleveland, Ohio 44135-3191

9. SPONSORING/MONITORING AGENCY NAME(S) AND ADDRESS(ES)

National Aeronautics and Space Administration

Washington, D.C. 20546-0001

10. SPONSORING/MONITORING AGENCY REPORT NUMBER

NASA TM-106869

ICOMP-95-5

AIAA-95-0681

11. SUPPLEMENTARY NOTES

Prepared for the 33rd Aerospace Sciences Meeting and Exhibit sponsored by the American Institute of Aeronautics and Astronautics, Reno, Nevada, January 9-12, 1995. S.H. Shih and D.R. Hixon, Institute for Computational Mechanics in Propulsion, Lewis Research Center, Cleveland, Ohio (work funded under NASA Cooperative Agreement NCC3-370); R.R. Mankbadi, NASA Lewis Research Center. ICOMP Program Director, Louis A. Povinelli, organization code 2600, (216) 433-5818.

12a. DISTRIBUTION/AVAILABILITY STATEMENT

12b. DISTRIBUTION CODE

Unclassified - Unlimited

Subject Category 34

This publication is available from the NASA Center for Aerospace Information, (301) 621-0390.

13. ABSTRACT (Maximum 200 words)

The unsteady structure of a supersonic jet is highly three dimensional, though the mean flow is axisymmetric. In simulating a circular jet, the centerline represents a computational boundary. As such, spurious modes can be generated near centerline, unless special attention is given to the behavior of the $3 \mathrm{D}$ structure near the centerline. Improper treatment of the dependent variables near the centerline results in the solution diverging or being suitable only for small amplitude excitation. With a careful treatment of the centerline formulation, no spurious mode is generated. The results show that a near linear disturbance growth is obtained, as the linear stability theory indicates. At high levels of excitation, nonlinear development of disturbances is evident and saturation is reached downstream.

\section{SUBJECT TERMS}

Unsteady; Large eddy simulation; Supersonic jet; Shear flow; Centerline 15. NUMBER OF PAGES

\begin{tabular}{|c|c|}
\hline 17. SECURITY CLASSIFICATION & $\begin{array}{c}\text { 18. SECURITY CLASSIFICATION } \\
\text { OF REPORT }\end{array}$ \\
$\begin{array}{c}\text { OF THIS PAGE } \\
\text { Unclassified }\end{array}$ & Unclassified
\end{tabular}

NSN 7540-01-280-5500
19. SECURITY CLASSIFICATION OF ABSTRACT

Unclassified
20. LIMITATION OF ABSTRACT 